\title{
Susceptibility of community associated methicillin resistant Staphylococcus aureus isolated from faeces to antiseptics
}

\author{
Ezekiel Olugbenga Akinkunmi and Adebayo Lamikanra
}

Department of Pharmaceutics, Obafemi Awolowo University, Ile-Ife, Nigeria

\begin{abstract}
Introduction: The study aimed to investigate the resistance of methicillin resistant Staphylococcus aureus (MRSA), an indicator used in hospitals but isolated from faecal samples of children in the community, to commonly used antibiotics and antiseptic agents.

Methodology: S. aureus isolates were identified by phenotypic and genotypic techniques such as biochemical tests and polymerase chain reaction. Antibiotic susceptibility was investigated using the disc diffusion technique while the agar dilution method was used to determine the minimum inhibitory concentration (MIC) of antiseptics.

Results: MRSA showed considerably higher resistance to other antibiotics than methicillin sensitive Staphylococcus aureus (MSSA). Twelve percent of the MSSA were susceptible to all the antibiotics studied while none of the MRSA had this property. A significant difference in susceptibility between MRSA and MSSA to the three antiseptic agents was observed as $68.8 \%, 75.0 \%$ and $100 \%$ of MRSA were less susceptible to benzalkonium chloride, chlorhexidine and cetrimide respectively, while $32.0 \%, 28.0 \%$ and $56.0 \%$ of MSSA respectively were less susceptible to these agents compared with $S$. aureus NCTC 6571 . Overall, the MICs for the antiseptics were 2-3 times greater in the MRSA than in the MSSA $(\mathrm{p}<0.001)$.

Conclusion: Results show that the concentration of antiseptics used in the prevention of the transmission of infectious agents may have to be raised to cope with the possible presence of MRSA in patients coming into hospital.
\end{abstract}

Key words: community associated methicillin resistant Staphylococcus aureus; faeces; susceptibility; antibiotics; antiseptics

J Infect Dev Ctries 2012; 6(4):317-324.

(Received 25 September 2010 - Accepted 06 July 2011)

Copyright (C) 2012 Akinkunmi and Lamikanra. This is an open-access article distributed under the Creative Commons Attribution License, which permits unrestricted use, distribution, and reproduction in any medium, provided the original work is properly cited

\section{Introduction}

The ability of Staphylococcus aureus to develop resistance to antibiotics has resulted in the emergence of methicillin resistant S. aureus (MRSA). MRSA has become a serious threat to hospitalized patients worldwide and it is now a challenge for public health practitioners [1]. Additionally, infections caused by community associated MRSA (CAMRSA) appear to be on the increase in both adults and children in various regions and countries of the world [2] while there are reports of a high rate of colonization of MRSA in healthy children [3]. This phenomenon is an indication of the accelerated spread of MRSA in the community and it has been shown to be as high as $60 \%$ within some communities [4].

Family members who are living with MRSA carriers have been shown to be in the greatest danger of MRSA transmission in any community $[4,5]$ and therefore should be targeted for protection. The concept of cleansing hands with an antiseptic agent probably emerged in the early $19^{\text {th }}$ century. Today, antiseptics are used in hygienic hand washes to reduce the transient microfloral on the hands, to reduce person-to-person transmission of microbes (e.g. MRSA), and to achieve surgical hand antisepsis in the hospital [6]. Hand-washing is considered an important tool in the control of nosocomial infections [7]. The effectiveness of this method, however, may be compromised by a decrease in the susceptibility of microorganisms to frequently used antiseptic agents, which may have to be used in higher concentrations to remain effective.

Antiseptic agents include various compounds with different chemical structures such as dyes, alcohols and surfactants [8]. Surface active agents such as chlorhexidine, benzalkonium chloride and cetyltrimethyl ammonium bromide (cetrimide), are commonly used antiseptic agents because of their relative nontoxicity to human tissues [6,9].

MRSA with decreased antiseptic susceptibility have been isolated from clinical samples and settings [10]. Although chlorhexidine, benzalkonium chloride and cetrimide are widely used, increased MICs for MRSA strains against these agents with positive cross-resistance to other antiseptics and antibiotics have been documented $[11,12,13]$.

Epidemiological information on antiseptic susceptibility is useful in the control of nosocomial 
and community associated infections. However, in Nigeria, there is little or no information about the susceptibility of nosocomial and community pathogens, such as MRSA, to antiseptics. The purpose of this study was to monitor the susceptibility of $S$. aureus isolated from faecal samples provided by apparently healthy children to three commonly used antiseptics in this environment and determine the possibility of any positive correlation between these agents and antibiotics.

\section{Methodology}

Sample collection

Three hundred stool samples were collected from children presenting for immunisation and treatment at five different community health centres and children attending four day-care centres in Ile-Ife, Nigeria, over a period of 6 months from January to June, 2006.

Isolation, identification and molecular characterisation of $\mathrm{S}$. aureus

Collected specimens were plated on mannitol salt agar (Biolab, Budapest, Hungary) and incubated at $37^{\circ} \mathrm{C}$ for $24-48$ hours. Pure colonies obtained were then subcultured onto fresh mannitol salt agar and blood agar. S. aureus were identified by colonial characteristics on blood agar and mannitol salt agar by Gram's stain reactions, and by biochemical tests including catalase, modified oxidase, alkaline phosphatase, and slide and tube coagulase tests [9]. The isolates were cryopreserved in cryovials (Nalgene, Rochester, NY, USA) and stored at $-20^{\circ} \mathrm{C}$.

$S$. aureus species were confirmed by polymerase chain reaction (PCR) amplification of thermostable nuclease gene (nuc) using the primers, nuc-F (5'GCGATTGATGGTGATACGGTT-3') and nuc-R (5'-AGCCAAGCCTTGACGAACTAAAGC-3') as described [14].

Bacterial DNA were extracted using the colony boiling method. Briefly, isolates were grown on nutrient agar at $35^{\circ} \mathrm{C}$ for $18-24$ hours. Two to three colonies of bacteria were picked using a sterile toothpick. These colonies were suspended in $100 \mu 1$ of nuclease free water in an eppendorf tube and boiled in a water bath at $100^{\circ} \mathrm{C}$ for 5 minutes to release the DNA and placed on ice for at least 5 minutes and then pulse centrifuged. The supernatant was used in the PCR reaction. $S$. aureus (MRSA) ATCC $^{\mathrm{R}} 43300$ was used for the PCR analysis as positive control while sterile distilled water was used as negative control.
PCR conditions for amplification of the $n u c$ gene comprised a predenaturation step of $95^{\circ} \mathrm{C}$ for 5 minutes, followed by 40 cycles of denaturation at $95^{\circ} \mathrm{C}$ for 30 seconds, annealing at $55^{\circ} \mathrm{C}$ for 30 seconds, and extension at $72^{\circ} \mathrm{C}$ for 1 minute, followed by a final extension for 5 minutes at $72^{\circ} \mathrm{C}$.

\section{Antibiotic susceptibility testing}

The susceptibilities of the $S$. aureus isolates against twelve commonly used antibiotics were tested by the disc diffusion method using inocula equivalent to 0.5 McFarland standard, prepared as described [15] on Mueller Hinton Agar plates (Remel, Lenexa, USA). The following antimicrobial agents at the indicated concentration were tested: chloramphenicol (CL) $30 \mu \mathrm{g} /$ disc, fusidic acid (FU) $50 \mu \mathrm{g} / \mathrm{disc}$, ciprofloxacin (CI) $10 \mu \mathrm{g} /$ disc, penicillin V (PV) $10 \mu \mathrm{g} / \mathrm{disc}$ cephadroxil (DX) $30 \mu \mathrm{g} / \mathrm{disc}$, erythromycin (EM) $15 \mu \mathrm{g} /$ disc, and tobramycin (TM) $30 \mu \mathrm{g} / \mathrm{disc}$ obtained from AB-Biodisc (Solna, Sweden); tetracycline (TE) $30 \mu \mathrm{g} /$ disc, co-trimoxazole (COT) $25 \mu \mathrm{g} / \mathrm{disc}$, and gentamicin (GEN) $30 \mu \mathrm{g} / \mathrm{disc}$ obtained from Abtek (Liverpool, England); and ampicillin (AMP) $10 \mu \mathrm{g} /$ disc and oxacillin (OX) $1 \mu \mathrm{g} / \mathrm{disc}$ obtained from Oxoid (Basingstoke, England). The diameters of inhibition zones were measured in millimeters and interpreted using the Progressive Diagnostics Manufacturers (PDM) Interpretive Charts (AB Biodisc, Solna, Sweden); this procedure agrees with the Clinical Laboratory Standard Institute (CLSI) [16] guidelines. S. aureus NCTC 6571 was used as control.

Oxacillin susceptibility testing using agar screening method

Oxacillin-salt agar (Mueller-Hinton agar containing $4 \% \quad \mathrm{NaCl}$ and $6 \mu \mathrm{g}$ oxacillin $/ \mathrm{ml}$ ), recommended by the CLSI [16], was used as an agar screening method for oxacillin susceptibility. Inoculation of the oxacillin-salt agar using inocula equivalent to $0.5 \mathrm{McF}$ arland standard was performed using a multipoint inoculator delivering approximately $1 \mu 1$ of the inoculum. The experiment was performed in duplicate. S. aureus (MRSA) ATCC $^{\mathrm{R}} 43300$ was used as the control.

\section{Antiseptics}

The following pure analytical grade antiseptics were used: benzalkonium chloride (BDH, Poole, England); cetrimide (Hopkin \& Williams, Essex, England); and chlorhexidine gluconate (Fluka Chemika, Buchs, Switzerland). 


\section{Determination of susceptibility to antiseptics}

Minimum inhibitory concentrations to the antiseptics; benzalkonium chloride $(0.5-32 \mu \mathrm{g} / \mathrm{ml})$, chlorhexidine gluconate $(0.5-32 \mu \mathrm{g} / \mathrm{ml})$ and cetrimide $(0.5-32 \mu \mathrm{g} / \mathrm{ml})$ were determined by the agar doubling dilution method according to CLSI guidelines [16]. The ranges of concentrations used for the agents were chosen following the reports of Noguchi et al. [10]. Plates containing $10 \mathrm{ml}$ of test medium incorporating doubling dilutions of antiseptic agents were prepared and dried. Plates containing no antiseptics were included as controls. An inoculum of the test organism equivalent to 0.5 McFarland was prepared and applied to the surface of antiseptic-containing and control agar plates using a multipoint inoculator. The lowest concentration of antiseptic inhibiting growth was considered the MIC. The experiments were performed in duplicate.

\section{Statistical analysis}

Chi-square test or the Fisher exact test was used in determining probabilities and level of significance. All hypotheses were considered significant if $\mathrm{p}<$ 0.05. The analysis was performed using SPSS Version 12 statistical software (IBM, Chicago, USA).

\section{Results}

Only 41 of the 300 stool samples examined yielded $S$. aureus isolates, as confirmed by the amplification of the $n u c$ gene, and of these, 16 $(39.0 \%)$ were found to be oxacillin resistant. Table 1 shows the antibiotic resistance profile of both MRSA and MSSA isolates obtained. The majority of MRSA isolates were resistant to most antibiotics; however less than $50 \%$ of isolates were resistant to fusidic acid, tobramycin, ciprofloxacin and gentamicin with resistance rates of $43.8 \%, 31.3 \%, 18.8 \%$ and $0 \%$ respectively. In addition to these agents, resistance to co-trimoxazole, chloramphenicol and tetracycline was lower than $50 \%$ in MSSA isolates.

The pattern of resistance in the MSSA and MRSA isolates is shown in Table 2. Three $(12.0 \%)$ of the MSSA isolates were completely susceptible to all antibiotics tested; however, none of the MRSA isolates were susceptible to all the antibiotics screened.

Table 3 shows the MICs of antiseptic agents against the $S$. aureus isolates. The MICs at which $50 \%$ and $90 \%$ of isolates were inhibited $\left(\mathrm{MIC}_{50}\right.$ and $\mathrm{MIC}_{90}$, respectively) by antiseptics for MRSA and MSSA were noted. $\mathrm{MIC}_{50}$ and $\mathrm{MIC}_{90}$ based on all the $S$. aureus isolates were also noted. Based on the
Table 1. Antibiotic resistance of MRSA and MSSA isolated cultured from faecal samples of children

\begin{tabular}{lcc}
\hline $\begin{array}{l}\text { Antimicrobial } \\
\text { tested }\end{array}$ & \multicolumn{2}{l}{ Resistance } \\
\cline { 2 - 3 } & $\begin{array}{l}\text { MRSA (n=16), } \\
\text { No. (\%) }\end{array}$ & $\begin{array}{l}\text { MSSA (n=25), } \\
\text { No. (\%) }\end{array}$ \\
\hline Penicillin V & $16(100.0)$ & $21(84.0)$ \\
Ampicillin & $15(93.8)$ & $21(84.0)$ \\
Cephadroxil & $14(87.5)$ & $18(72.0)$ \\
Erythromycin & $14(87.5)$ & $13(52.0)$ \\
Co-trimoxazole & $10(62.5)$ & $9(36.0)$ \\
Chloramphenicol & $8(50.0)$ & $8(32.0)$ \\
Tetracycline & $9(56.3)$ & $7(28.0)$ \\
Fusidic acid & $7(43.8)$ & $2(8.0)$ \\
Ciprofloxacin & $3(18.8)$ & $2(8.0)$ \\
Tobramycin & $5(31.3)$ & $1(4.0)$ \\
Gentamicin & $0(0.0)$ & $0(0.0)$ \\
\hline
\end{tabular}

MICs of antiseptic agents obtained against the reference strain, $S$. aureus NCTC 6571 , the criteria for susceptibility to each of benzalkonium chloride, chorhexidine and cetrimide in $S$. aureus were defined as MIC $\leq 4 \mu \mathrm{g} / \mathrm{ml}$ (Table 3). Among MRSA isolates, $68.8 \%, 75.0 \%$ and $100 \%$ were more resistant to benzalkonium chloride, chlorhexidine and cetrimide respectively than $S$. aureus NCTC 6571. Among the MSSA isolates, $32.0 \%, 28.0 \%$ and $56.0 \%$ were more resistant to benzalkonium chloride, chlorhexidine gluconate and cetrimide respectively than $S$. aureus NCTC 6571. The susceptibility of MRSA and MSSA to antiseptics and the significance are shown in Table 4.

\section{Discussion}

The essential role of antiseptics in reducing the transient microflora of the hands and reducing person-to-person transmission of antibiotic resistant and pathogenic microbes such as MRSA, coupled with the reports of microbial resistance to these agents, underscores the need to assess the susceptibility of these microbes to antiseptic agents. Despite the importance of such assessment, the susceptibility of MRSA to the antiseptic agents investigated in this study, chlorhexidine, benzalkonium chloride and cetrimide, has not been reported previously in the study environment.

In the present study, the susceptibility of $S$. aureus MRSA and MSSA isolates from faecal samples of children to various commonly used antibiotics and antiseptic agents was examined. $S$. aureus has established itself as a nosocomial and 
Table 2. Resistance patterns of the MSSA and MRSA isolates

\begin{tabular}{|c|c|}
\hline $\begin{array}{l}\text { MSSA } \\
\text { Strains }\end{array}$ & Resistance patterns \\
\hline$\overline{\mathrm{A} 10 \mathrm{~A}}$ & - \\
\hline A123A & - \\
\hline A264B & - \\
\hline A69B & PV, AMP \\
\hline A132B & PV, AMP \\
\hline A202A & AMP, COT \\
\hline A207A & PV, AMP, DX \\
\hline A255A & PV, AMP, DX \\
\hline A59C & PV, AMP, DX, EM \\
\hline A94D & PV, EM, COT, CI \\
\hline A157B & PV, AMP, DX, EM \\
\hline A224B & PV, AMP, DX, CL \\
\hline A249A & PV, AMP, DX, CL \\
\hline A291B & PV, AMP, DX, TE \\
\hline A250A & PV, AMP, DX, EM, COT \\
\hline $\mathrm{A} 275 \mathrm{~A}$ & PV, AMP, DX, CL, FU \\
\hline A283B & PV, AMP, DX, CL, EM \\
\hline A284A & PV, AMP, DX, EM, COT \\
\hline A118B & PV, AMP, DX, TE, EM, COT \\
\hline A159A & PV, AMP, DX, EM, COT, CI, \\
\hline A180B & PV, AMP, DX, TE, EM, CL, \\
\hline A251A & PV, AMP, DX, TE, EM, CL \\
\hline A47B & PV, AMP, DX, TE, EM, CL, COT \\
\hline A91B & PV, AMP, DX, TE, EM, CL, COT \\
\hline A $285 \mathrm{~A}$ & PV, AMP, DX, TE, EM, COT, FU, TM \\
\hline $\begin{array}{l}\text { MRSA } \\
\text { Strains }\end{array}$ & Resistance patterns \\
\hline A49C & $\mathrm{PV}, \mathrm{OX}$ \\
\hline A293A & PV, AMP, CL, OX \\
\hline A196C & PV, AMP, DX, EM, COT, OX \\
\hline $\mathrm{A} 205 \mathrm{~A}$ & PV, AMP, DX, EM, COT, OX \\
\hline A88A & PV, AMP, DX, EM, COT, FU, OX \\
\hline A225B & PV, AMP, DX, EM, COT, FU, OX \\
\hline A276A & PV, AMP, DX, TE, EM, CL, OX \\
\hline $\mathrm{A} 32 \mathrm{~A}$ & PV, AMP, DX, TE, EM, COT, CI, OX \\
\hline $\mathrm{A} 75 \mathrm{C}$ & PV, AMP, DX, EM, CL, FU, CI, OX \\
\hline A144A & PV, AMP, DX, TE, EM, COT, FU, OX \\
\hline A244B & PV, AMP, DX, TE, EM, CL, COT, OX \\
\hline $\mathrm{A} 43 \mathrm{~A}$ & PV, AMP, DX, TE, EM, CL, COT, TM, OX \\
\hline A120A & PV, AMP, DX, TE, EM, CL, FU, TM, OX \\
\hline A254B & PV, AMP, DX, TE, EM, CL, COT, TM, OX \\
\hline A290B & PV, AMP, DX, TE, EM, CL, FU, TM, OX \\
\hline A9A & PV, AMP, DX, TE, EM, COT, FU, TM, CI, OX \\
\hline
\end{tabular}

TE: Tetracycline; COT: Cotrimoxazole; CL: chloramohenicol; FU: Fusidic acid; CI: Ciprofloxacin; PV: Penicillin V; AMP: Ampicillin; DX: Cephadroxil; EM: Erythromycin; TM: Tobramycin; OX: Oxacillin. 
Table 3. Antiseptic susceptibility of methicillin-resistant and methicillin-sensitive $S$. aureus isolates

\begin{tabular}{llll}
\hline Staphylococci isolates $(\mathbf{N o})$ & Antimicrobial agents & $\mathbf{M I C}^{\mathbf{a}}(\boldsymbol{\mu g} / \mathbf{m l})$ \\
\hline \multirow{2}{*}{ MRSA $(\mathrm{n}=16)$} & & $\mathbf{M I C}_{\mathbf{5 0}}$ & $\mathbf{M I C}_{\mathbf{9 0}}$ \\
\cline { 2 - 3 } & Benzalkonium chloride & 32 & $>32$ \\
& Chlorhexidine & 32 & $>32$ \\
& Cetrimide & $>32$ & $>32$ \\
MSSA $(\mathrm{n}=25)$ & Benzalkonium chloride & 4 & 16 \\
& Chlorhexidine & 2 & 32 \\
& Cetrimide & 16 & 32 \\
Total S. aureus $(\mathrm{n}=41)$ & & & 32 \\
& Benzalkonium chloride & 8 & $>32$
\end{tabular}

${ }^{\mathrm{a}} \mathrm{MIC}_{50}$ and $\mathrm{MIC}_{90},=\mathrm{MICs}$ at which 50 and $90 \%$ of isolates, respectively, were inhibited. MIC of each antiseptic and antibiotic for the reference strain, S. aureus $\mathrm{NCTC} 6571$, are as follows: BKC, $4 \mu \mathrm{g} / \mathrm{ml}$; CHG, $4 \mu \mathrm{g} / \mathrm{ml}$; CTM, $4 \mu \mathrm{g} / \mathrm{ml}$.

Table 4. Susceptibility of MRSA and MSSA to antiseptics

\begin{tabular}{llccc}
\hline \multirow{2}{*}{ Antiseptics } & \multicolumn{4}{l}{ Isolates with reduced susceptibility, Number $(\%$ of total $)$ : } \\
\hline & MRSA & MSSA & Total & p-value $^{\mathrm{a}}$ \\
\cline { 2 - 5 } Benzalkonium chloride & 16 & 25 & 41 & \\
Chlorhexidine & $11(68.8)$ & $8(32.0)$ & $19(46.3)$ & $1.95 \times 10^{-7}$ \\
Cetrimide & $12(75.0)$ & $7(28.0)$ & $19(46.3)$ & $2.94 \times 10^{-11}$ \\
& $16(100.0)$ & $14(56.0)$ & $30(73.2)$ & $5.88 \times 10^{-14}$ \\
\hline
\end{tabular}

community pathogen with a very high potential to acquire resistance to antimicrobial agents and this has resulted in the development of methicillin resistant $S$. aureus which is increasingly associated with serious infections globally [1]. In addition to this, MRSA and especially CAMRSA, are increasingly associated with multidrug resistance including resistance to antiseptics and disinfectants [10,17].

Generally, in this study, resistance to the tested antimicrobial agents by $S$. aureus isolates was high. Earlier studies on the antibiotics resistance of $S$. aureus in subjects in Ile-Ife have shown that the incidence of resistance of $S$. aureus to antibiotics was increasing even within the hospital environment where some measure of control might be expected $[18,19]$. One of these studies reported 100\% penicillin resistance for $S$. aureus [19]. The present study has shown that although a few strains of CAMSSA are still susceptible to penicillin, all the CAMRSA isolates obtained were resistant to this antibiotic. In addition, most of these isolates were resistant to other antibiotic families that were tested.
Methicillin resistance in MRSA is related to a chromosomal mecA gene that specifies the production of an abnormal penicillin binding protein, PBP2a or PBP2' [1]. The mecA gene complex also contains insertion sites for plasmids and transposons that facilitate acquisition of resistance to other antibiotics and other antimicrobial agents such as disinfectants and antiseptics [1].

While the majority of CAMSSA isolates were still susceptible to cotrimoxazole, tetracycline, chloramphenicol, fusidic acid and ciprofloxacin (ranging from 60-96\%), among this group of antimicrobial agents, CAMRSA were only susceptible to ciprofloxacin. The high percentage of resistant CAMRSA observed in this study is of great concern as it shows that some children within the community are carriers of multidrug resistant organisms, a scenario which may have large implications for public health. Given this situation, it is important to put measures in place to curtail the transmission of these organisms from person to person. One of the ways of achieving this objective is 
to promote the use of antiseptics with the capacity to reduce the concentration of microbes present.

Cationic antiseptic agents such as quaternary ammonium compounds and chlorhexidine are commonly used for disinfection of skin and hands [6]. These antiseptic compounds are, however, only useful in situations in which target organisms are susceptible at relatively low concentrations.

The criteria for susceptibility to each antiseptic agent were defined by the MICs of the selected antiseptics to the reference strain, $S$. aureus NCTC 6517. These criteria were previously used by Noguchi et al. [10] while examining the susceptibility to antiseptic agents and distribution of antiseptic - resistance genes in MRSA collected in Asian countries between 1998 and 1999. Based on our results, CAMRSA isolates had higher MIC values than CAMSSA isolates, ranging from a twofold difference for benzalkonium chloride and cetrimide, and a threefold difference for chlorhexidine.

Several researchers have suggested a link between the susceptibility of organisms to antibiotics and other antimicrobial agents such as disinfectants and antiseptics [20]. Levy [21] demonstrated this scenario for benzalkonium chloride. He noted that resistance to beta-lactam antibiotics among MRSA isolates was exclusively associated with decreased susceptibility to benzalkonium chloride; this also matched the susceptibility pattern of the MRSA SCCmec-type IV isolates found within the community setting. Other investigators found clinical MRSA isolates to have slightly decreased susceptibility relative to susceptible isolates to a range of biocides that included chlorhexidine, cetyl pyridinium chloride, benzalkonium chloride, cetrimide, hypochlorite, parahydroxybenzoates, betadine and triclosan $[10,13,17]$.

Although the tests conducted in this study did not necessarily reflect all the conditions under which the antiseptics are put to use in the homes and in the hospitals, the level of decreased susceptibility exhibited by the MRSA isolates suggests that faecal MRSA $S$. aureus and to some extent MSSA isolates could survive in homes and even in the hospital environment where these agents are used for disinfection purposes.

It can therefore be suggested that higher concentrations or increased contact times of effective antiseptics should be used for the disinfection of skin as well as general disinfection to ensure the elimination of resistant strains and the prevention of cross-infection of family members at home, other children and the care givers at child care centres, and patients and hospital personnel. The use of an elevated concentration of antiseptic will help in eliminating multiresistant nosocomial and community pathogens and may thus prevent infection [22].

In conclusion, the results of this study show that there is a direct relationship between resistance to methicillin and a significantly decreased susceptibility to benzalkonium chloride, cetrimide and chlorhexidine even if this decreased susceptibility may not be enough to abrogate the effectiveness of these agents at the concentrations of their intended use. However, the decrease in susceptibility highlighted in this study may be sufficient to warrant the use of these agents at higher concentration and for longer contact periods than they are recommended for general use. It must be recognized that the continued usefulness of antiseptics in reducing the transmission of MRSA and indeed other pathogens within both the community and the hospital environment is of crucial importance and must therefore be a subject for continuous study.

\section{Acknowledgements}

We thank the management and staff of the institutions where samples were collected for support and technical assistance, the Central Science Laboratory, Obafemi Awolowo University (OAU), Ile-Ife, and Dr I. N. Okeke of Haverford College, USA, for materials used for molecular studies and for helpful discussions.

\section{References}

1. Chamber HF (2005) Community-associated MRSAresistance and virulence converge. New Engl J Med 352: 1485-1487.

2. Donnio PY, Fe'vrier F, Bifani P, Dehem M, Kerve'gant C, Wilhelm N, Gautier Lerestif AL, Lafforgue N, Cormier M, the MR-MSSA Study Group of the Colle'ge de Bacte'riologie-Virologie-Hygie'ne des Hôpitaux de France, Coustumier AL (2007) Molecular and epidemiological evidence for spread of multiresistant methicillin-susceptible Staphylococcus aureus strains in Hospitals. Antimicrob Agent Chemother 51: 4342-4350.

3. Huang YC, Hwang KP, Chen PY, Chen CJ, Lin TY (2007) Prevalence of methicillin-resistant Staphylococcus aureus nasal colonization among Taiwanese children in 2005 and 2006. J Clin Microbiol 45: 3992-3995.

4. Matsumoto K, Hohashi N, Sugishita C (2001) A study on the transmission of MRSA among the family members including clients of visiting nurse and related infection control. Nippon Koshll Eisei Zasshi 48: 190-199.

5. Huijsdens XW, van Santen-Verheuvel MG, Spalburg E, Heck MEOC, Pluister GN, Eijkelkamp BA, de Neeling AJ, Wannet WJB (2006) Multiple cases of familial transmission 
of community-acquired methicillin-resistant Staphylococcus aureus . J Clin Microbiol 44: 2994-2996.

6. Boyce JM and Pittet D (2002) Guideline for hand hygiene in health-care settings. Recommendations of the Healthcare Infection Control Practices Advisory Committee and the HICPAC/SHEA/APIC/IDSA Hand Hygiene Task Force. Society for Healthcare Epidemiology of America/Association for Professionals in Infection Control/Infectious Diseases Society of America. Morbid Mortal Wkly Rep Recomm Rep 51, 1-4.

7. Nystrom B (1994) Impact of handwashing on mortality in intensive care: examination of the evidence. Infect Control Hosp Epidemiol 15: 435-436.

8. Mcdonnell G and Russell AD (1999) Antiseptics and disinfectants: activity, action, and resistance. Clin Microbiol Rev 12: 147-179.

9. Mengistu Y, Ergie W, Bellete B (1999) In vitro suceptibility of staphylococci to chlorhexidine and antibiotics. Ethiop $\mathbf{J}$ Health Dev 13: 223-28.

10. Noguchi N, Suwa J, Narui K, Sasatsu M, Ito T, Hiramatsu K, Song J-H (2005) Susceptibilities to antiseptic agents and distribution of antiseptic-resistance genes qacA/B and smr of methicillin-resistant Staphylococcus aureus isolated in Asia during 1998 and 1999. J Med Microbiol 54: 557-565.

11. Brumfitt W, Dixson S, Hamilton-Miller JMT (1985) Resistance to antiseptics in methicillin and gentamicin resistant Staphylococcus aureus. Lancet i: 1442-1443.

12. Al-Masaudi SB, Russell AD, Day MJ (1991) Comparative sensitivity to antibiotics and biocides of methicillin-resistant Staphylococcus aureus strains isolated from Saudi Arabia and Great Britain. J Appl Bacteriol 71: 331-338.

13. Irizzary L, Merlin T, Rupp J, Griffith J (1998) Reduced susceptibility of methicillin resistant Staphylococcus aureus to cetylpyridinium chloride and chlorhexidine. Chemother 42: 248-252.

14. Brakstad OG, Aasbakk K, Maeland JA (1992) Detection of Staphylococcus aureus by polymerase chain reaction amplification of the nuc gene. J Clin Microbiol 30: 16541660 .
15. Felten A, Grandry B, Lagrange PH, Casin I (2002) Evaluation of three techniques for detection of low-level methicillin-resistant Staphylococcus aureus (MRSA): a disk diffusion method with cefoxitin and moxalactam, the Vitek 2 System, and the MRSA-screen latex agglutination test. J Clin Microbiol 40: 2766-2771.

16. Clinical and Laboratory Standards Institute (2006) Performance standards for antimicrobial susceptibility testing; 16th informational supplement. M100-S16. Clinical and Laboratory Standards Institute, Wayne, $\mathrm{Pa}$.

17. Suller MTE and Russell AD (1999) Antibiotic and biocide resistance in methicillin resistant Staphylococcus aureus and vancomycin-resistant Enterococcus. J Hosp Infect 43: 281291.

18. Lamikanra A and Olusanya OI (1988) A long-term study of the nasal carriage of Staphylococcus aureus in healthy Nigerian students. Transactions of the Royal Society of tropical medicine and hygiene 82: 500-502.

19. Ako-nai KA, Ogunniyi AD, Lamikanra A, Torimiro SEA (1991) The characterisation of clinical isolates of Staphylococcus aureus in Ile-Ife, Nigeria. J Med Microbiol 34: 109-112.

20. Aiello AE and Larson E (2003) Antibacterial cleaning and hygiene products as an emerging risk factor for antimicrobial drug resistance in the community. Lancet Infect Dis 3: 501-506.

21. Levy SB (2000) Antibiotic and antiseptic resistance: impact on public health. Pediatr Infect Dis J 19: S120-S122.

\section{Corresponding author}

Ezekiel Olugbenga Akinkunmi

Address: Department of Pharmaceutics

Faculty of Pharmacy

Obafemi Awolowo University

Ile-Ife, Nigeria

Telephone: +234-803-654-8975

Email: eoakinmi@oauife.edu.ng

Conflict of interests: No conflict of interests is declared. 